\title{
BMJ Open Rare but heard: using asynchronous virtual focus groups, interviews and roundtable discussions to create a personalised psychological intervention for primary sclerosing cholangitis: a protocol
}

\author{
Veronica Ranieri (D) , ${ }^{1,2}$ Eilis Kennedy, ${ }^{1}$ Martine Walmsley, ${ }^{3}$ Doug Thorburn, ${ }^{4}$ \\ Kathy McKay ${ }^{1}$
}

To cite: Ranieri V, Kennedy E, Walmsley M, et al. Rare but heard: using asynchronous virtual focus groups, interviews and roundtable discussions to create a personalised psychological intervention for primary sclerosing cholangitis: a protocol. BMJ Open 2019;9:e031417. doi:10.1136/ bmjopen-2019-031417

- Prepublication history for this paper is available online. To view these files, please visit the journal online (http://dx.doi. org/10.1136/bmjopen-2019031417).

Received 02 May 2019 Revised 02 September 2019 Accepted 17 September 2019

Check for updates

(c) Author(s) (or their employer(s)) 2019. Re-use permitted under CC BY-NC. No commercial re-use. See rights and permissions. Published by BMJ.

${ }^{1}$ Research and Development, Tavistock and Portman NHS Foundation Trust, London, UK ${ }^{2}$ Science and Technology Studies, University College London, London, UK

${ }^{3}$ PSC Support, Oxford, UK

${ }^{4}$ Department of Hepatology, Royal Free London NHS Trust, London, UK

Correspondence to Dr Veronica Ranieri; v.ranieri@ucl.ac.uk

\section{ABSTRACT}

Introduction Primary sclerosing cholangitis (PSC) is a rare and chronic disease characterised by inflammation and fibrosis of the liver's bile ducts. There is no known cause or cure for the illness, which often progresses to end-stage liver disease requiring liver transplantation. Symptoms of PSC can be very burdensome on those living with the illness, leading to restrictions in daily living, as well as a greater risk of colorectal and biliary tract cancers. Limited voices from lived experience suggest that living with PSC can cause considerable psychological distress. This study, therefore, aims to explore how the illness impacts the psychological well-being of those living with the illness, and those supporting them. It also aims to create a personalised psychological intervention to support all groups.

Methods and analysis This project will take a layered qualitative approach to understanding the ways in which people experience living with PSC within their day-today lives. There will be two stages to this study, which will pilot a unique methodological process using online resources. The first stage will consist of asynchronous virtual focus groups (AVFGs) with those living with PSC and those who provide support for those diagnosed with PSC, and narrative interviews with both groups and health professionals. Both the AVFGs and the narrative interviews will be analysed using thematic narrative analysis. The second stage will comprise a roundtable discussion where the researchers and health professionals will devise a personalised psychological intervention to help to support those living with PSC and their supporters. The study duration is expected to be 18 months.

Ethics and dissemination The proposed study has been approved by the UK Health Research Authority and London-Queen Square Research Ethics Committee as application 18/L0/1075. Results from the AVFGs and the narrative interviews will be submitted for peerreviewed publication. The findings of the study will also be presented nationally to PSC and medical communities, and a summary of the findings will be shared with participants.
Strengths and limitations of this study

- This study will employ innovative qualitative research methods, such as the use of asynchronous virtual focus groups (AVFGs), to capture the lived experiences of those with a rare illness spread over a large geographic space who would not be able to participate in a study like this otherwise.

- A triangulated perspective will be used to explore the experiences of those living with primary sclerosing cholangitis, caregivers and health professionals, with a view to improving the support provided to all.

- A personalised psychological intervention, which can be tailored to fit individuals' personal circumstances, will be created in response to analysing these experiences.

- However, involvement in AVFGs is dependent on participants' literacy, computer literacy and access to technology.

- Although online qualitative approaches can target difficult-to-access populations, they cannot replace face-to-face human contact and interaction.

\section{INTRODUCTION}

Primary sclerosing cholangitis (PSC) is a rare and chronic disease characterised by the narrowing and blockage of the liver's bile ducts from inflammation and fibrosis. ${ }^{1}$ Recent estimates suggest that the incidence of PSC is 0.68 per 100000 person-years. ${ }^{2}$ It disproportionately affects men, and symptoms include jaundice, fatigue and itching, among others. ${ }^{13}$ However, these symptoms vary: some people are asymptomatic when first diagnosed, whereas others have a significant symptom burden. ${ }^{4}$ In addition to this variation in symptoms, the speed and aggressiveness with which the disease can progress 
may differ between individuals, with little indication from the literature as to why. ${ }^{1}$ Furthermore, there is no curative treatment for PSC, with liver transplant offered as an option for those reaching end-stage liver disease and those living with the illness displaying a greater incidence of colorectal and biliary tract cancers in addition to other complications. ${ }^{5} 6$

In the absence of curative treatment, recent research has begun to examine the impact of living with PSC on individuals' psychological well-being. ${ }^{7}$ Similar to other long-term physical illnesses, individuals living with PSC tend to report a greater number of depressive symptoms, and a greater level of impairments pertaining to quality of life and well-being compared with the general population. ${ }^{7-9}$ Voices from lived experience reveal that the uncertainty with which the disease may progress to an eventual need for a liver transplant, and whether and when that transplant may be possible, can lead to considerable distress and anxiety regarding the future. ${ }^{10} 11$ In addition to such distress, individuals with PSC also report feeling socially isolated due to the rarity of the illness. ${ }^{8}{ }^{12}$ Consequentially, such difficulties emphasise a need for access to emotional support that addresses this population's unmet needs and reinstalls hope in the future. $^{10}$

A need for greater access to support is likely also felt by those supporting individuals living with PSC, whether informally or formally. ${ }^{7}$ Partners and families supporting those living with PSC may need to adjust and prepare for caring for a serious illness. Due to the rarity of the illness, few healthcare professionals will have experience of how PSC can present and progress, and so treating the presenting individual may result in unexpected challenges for them, especially in finding appropriate and effective pathways of care for their patient. ${ }^{11}$ In light of these difficulties, the proposed study will aim to understand and chronicle the impact of PSC on the psychological well-being of those diagnosed with it and their caregivers, and on healthcare professionals' ability to support both. The study will seek to narrate the experiences of various people impacted by a PSC diagnosis, with a view to creating a personalised psychological intervention for all.

\section{Research questions}

1. What are the experiences of people living with PSC in terms of their mental health and emotional well-being?

2. What are the experiences of partners and family members of people living with PSC in terms of their mental health and well-being?

3. What are the experiences of healthcare professionals in working with people living with PSC and finding appropriate and effective pathways of care?

4. What would a framework of 'Personalised Psychological Intervention for PSC' for people with PSC and their partners and families look like?

\section{Objectives}

1. To explore the experiences of people living with PSC in terms of their mental health and emotional well-being.

2. To explore the experiences of family members and partners of people living with PSC in terms of their mental health and emotional well-being.

3. To explore the experiences of health professionals working with people living with PSC and finding appropriate and effective pathways of care.

4. To develop a framework of a 'Personalised Psychological Intervention for PSC'.

\section{METHODS AND ANALYSIS \\ Procedure}

This project will take a layered qualitative approach to understanding the ways in which people experience living with PSC within their day-to-day lives. There will be two stages to this study, which will also pilot a unique methodological process using online resources. The study duration is expected to be 18 months.

\section{Stage 1 answers research questions $1-3$, and will comprise of}

1. Asynchronous virtual focus groups (AVFGs) (research questions 1 and 2)

AVFGs are focus groups held online over a time period that is longer than a usual focus group, usually one question per week over a period of several weeks. Similar to a traditional focus group, they still have set time limits and the ability for participants to engage with each other. They have been previously used successfully with vulnerable and diverse populations. ${ }^{13}$ As PSC is so rare, conducting face-to-face research is difficult, so AVFGs are a good way of accessing as wide a group as possible. ${ }^{14}$

Separate AVFGs will be conducted with both people living with PSC and family members to allow participants to be as open and honest in their responses as they wish. AVFGs will be held on a virtual learning environment (VLE) platform and will be limited to 6-9 participants per group. Due to their online nature, more than one AVFG will be held at the same time. Participants will be asked to answer a different question each week for 4 weeks, relating to their experiences of receiving a diagnosis of PSC, their experiences with healthcare professionals, what helps or hinders their well-being and what advice they would share with someone recently diagnosed with PSC. One question will be posed per week and the participants can post as many responses as they wish each week; both in response to the question posed and what other responders have said. After a week, the previous question will be closed and the next one will be opened; this will be clearly stated. In this way, participants can respond when it suits them, with the responses being as long or as short as they feel comfortable with. The AVFGs will be moderated two times per day by researchers KM and VR to check the content of what is posted by participants. Participants 
will be asked to follow a series of common 'netiquette' guidelines, for instance, asking participants to respect other participants' points of view and refraining from using all caps. Should the moderators see evidence of severe psychological distress, researchers KM and VR will make direct contact with participants and redirect them towards the appropriate support. Every participant will also have online access to clear contact details for the research team and helplines (ie, Samaritans) for the duration of the study.

2. Narrative interviews (telephone/online/face-to-facemore in-depth experience) (answers research questions 1-3)

Narrative interviews are crucial to unpacking the themes identified from the AVFGs to inform the development of a personalised psychological intervention. Narrative interviews will be conducted with:

a. A purposive sample of participants living with PSC and family members who participated in the AVFGs.

b. Healthcare professionals recruited either via PSC Support or from asking those living with PSC who took part in AVFGs if they are willing to provide the name and contact details of their healthcare practitioner. As PSC is uncommon, it is expected that not every healthcare practitioner will be experienced in treating it, which can lead to feelings of stress when trying to determine the best pathway of care for their patient. Healthcare professionals will not have access to any data disclosed during interviews and focus groups with participants who have PSC, and vice versa.

Stage 2 answers research question 4 , and is based on the findings from stage 1

Practitioners from hepatology, gastroenterology and mental health will be invited to a presentation of the themes and narratives uncovered from the data in stage 1. Following this presentation, they will be asked to partake in an audio-recorded roundtable discussion on the findings of the focus groups and interviews. From these findings, they will be asked to help to co-construct a personalised psychological intervention. After the completion of this roundtable, the researchers will write-up the conclusions of the discussion and will send these to the practitioners for confirmation.

\section{Sample}

Participants will consist of those with lived experience of PSC, family members/partners/carers of people living with PSC and health professionals who have experience working with people living with PSC. This study aims to empower those living with PSC and their loved ones and those who care for them to make their voices heard. As such, we are keeping our inclusion criteria general so as to include as many people who would like to participate as possible. Participants must:

1. Be aged 18 years or more.

2. Be UK residents.
3. Self-identify as someone living with PSC or a partner, family member or carer of a person living with PSC, or a health professional working with people living with PSC.

Due to the rarity of this disease and the lack of extant research exploring experiences of PSC, it is difficult to specify the size of our sample. The study will aim to include a range of 37-62 participants overall. This range acknowledges that recruitment of individuals with PSC may be challenging given the rarity of the illness, but also ensures enough participation for data saturation. For the AVFGs, we aim to run 2-4 focus groups consisting of 6-9 participants in each. This will include 1-2 focus groups of people living with PSC and 1-2 focus groups of partners and family members of people living with PSC. From these, we plan on purposively sampling up to 10 individuals to take part in narrative interviews. Additionally, we aim to interview 5-10 health professionals.

\section{Setting}

Participants will be recruited using the PSC Support network. PSC Support is a charity that plays a key role in connecting people living with PSC to each other and wider PSC communities, as well as providing access to support and information. PSC Support will promote the study, as well as guide us on the appropriateness and relevance of the study design. The study will be advertised via PSC Support's media presence (ie, Facebook and Twitter). All interested people can contact the researchers (KM and VR) directly to get more information about the study. All study-specific activities (eg, recruitment, etc) will be undertaken by the researchers who are based in the Tavistock and Portman NHS Foundation Trust. As PSC is a rare disease, participants will be recruited from across the UK to allow greater participation.

AVFGs will be hosted on an existing VLE (ie, Moodle or Blackboard). ${ }^{15} 16$ VLEs are suitable for collecting this type of data as they enable researchers to host focus groups on their own custom-made site which is secure, confidential and a safe environment for research participants. ${ }^{17}$ For example, this allows the researchers to create ground rules for discussion which detail what style, tone and content of messages would be appropriate for discussions to ensure the well-being of all participants. ${ }^{17}$ AVFGs also allow the researchers to access participants regardless of location, which is likely to be an obstacle with rare diseases, such as PSC. When the AVFGs are ongoing, the researchers will check the content two times per day to ensure safety for all parties involved.

All interviews are expected to last a maximum of 1 hour. Many healthcare professionals face considerable time constraints in their workplace, so the interview will be constructed to take as little time as possible. Participation is entirely voluntary. Given the potential distance of participants, these will be conducted over the phone or via Telemedicine, depending on the person's preference. The opportunity to talk face-to-face or via email will be offered to local participants should they prefer to do so. 
Participants will be asked to give verbal audio-recorded consent and consent to their interview being audio-recorded. The questions within the interview will focus on similar themes as the AVFGs, but the narrative interview format will allow for a deeper exploration of people's experiences. Should any participant become distressed during the interview, they will be asked if they would like to discontinue the interview or the recording. At the conclusion of each interview, the researcher will debrief with the participant to check how they are feeling and offer contact details for helplines if needed.

\section{Analysis}

The data collected from stage 1 will be transcribed, coded and de-identified, and then analysed thematically and narratively using NVivo as the data management system. ${ }^{18}$ The first researcher (VR) will go through the transcripts from the AVFGs and the different interviews and analyse these using thematic narrative analysis. This will involve comparing themes and narratives both chronologically and between the three different groups (people with PSC, partners and family members of people with PSC and healthcare professionals). Themes and narratives will be double-checked by a second researcher (KM), and any disagreements will be discussed and resolved between the two researchers.

Findings will then be taken to the PSC Support Study Steering Committee to discuss in terms of what has been found, and whether anything has been missed by the researchers and would be an important inclusion in terms of creating the personalised psychological intervention framework. Following this, the group of gastroenterology, hepatology and mental health professionals will then be invited to attend a face-to-face roundtable meeting to discuss ideas and issues that could ground a 'Personalised Psychological Intervention for PSC' framework of recommendations, in conjunction with those with lived experience. This innovative framework of recommendations could help tailor interventions to not only the individuals diagnosed with PSC but also their partners and families, to ensure that they received the best support for their physical and mental health. Further, although the details would be personalised to each individual depending on their symptoms and severity, the framework of these interventions could then be shared to the wider healthcare professional community, so they would be better prepared to support a patient diagnosed with PSC. Again, this framework will then be shared with the PSC Support steering group to ensure that it is pragmatic and practical in terms of what people with PSC need in terms of care and support in their everyday lives.

\section{Patient and public involvement}

The study was co-designed with patient and public involvement from PSC Support, and will be overseen by a steering committee composed of individuals living with PSC or caring for relatives living with PSC. The study will be advertised by PSC Support and its findings will be evaluated and disseminated by members of PSC Support.

\section{ETHICS AND DISSEMINATION}

\section{Informed consent}

Informed consent will be obtained for each participant. All participants will be provided with an information sheet indicating the purpose of the study and will have an opportunity to ask questions. Consent for participants partaking in the AVFGs will be recorded online. Formal consent will also be recorded for all those taking part in narrative interviews. Should interviews be carried out over telephone or via Telemedicine, participants will be asked to give verbal consent, which will be audio-recorded. Signed and written consent will be sought from those taking part in face-to-face or email interviews. Consent from all members taking part in a roundtable discussion will be audio-recorded.

\section{Data protection and patient confidentiality}

Information provided in confidence will be anonymised and not be used or disclosed in a form that might identify the participant without his or her express written consent. Participants will be given the opportunity to choose their own pseudonym and these will be held in password-protected databases on an encrypted National Health Service (NHS) server at the Tavistock and Portman NHS Foundation Trust. Only the chief investigator and researchers will have access to it, and only anonymised data will be shared with anyone else. Only participants' unique pseudonyms will be linked to the raw data collected (eg, audio-recordings). Audio files will be identified by pseudocodes; however, it is possible that names of people and/or places may be mentioned during the interviews. All digital data files (including audio files) will be assigned a pseudocode and uploaded onto a secure NHS server as soon as possible after data are collected, and the files will be deleted from the memory disk or hard drive. Memory disks and hard drives containing data will be held in locked cabinets if it is not possible to upload the files immediately following data collection. Data will be uploaded onto the secure server, only accessible by authorised researchers, and will be transferred to a trusted transcription company using secure data transfer methods. All members of the research team will be reminded of their duty to observe the NHS code of ethics regarding patient confidentiality.

\section{Dissemination}

The findings of the study will be submitted for publication in peer-reviewed journals, and will be presented nationally. All intellectual property rights relating to the data arising from the study shall be held by the Tavistock and Portman NHS Foundation Trust. Participants who consent to receive the study findings will be sent an electronic or hard copy interim report and a final lay summary of the findings, depending on their preferred method of communication. They will also be provided 
with the details of where to access the online publication of the full study report.

Contributors EK and KM constructed the protocol. KM and VR drafted this protocol article. It was subsequently reviewed by all the authors (EK, DT and MW).

Funding This work was supported by PSC Support (grant number 220318VR). Competing interests None declared.

Patient consent for publication Not required.

Ethics approval This study was approved by the Health Research Authority and received a Favourable Opinion from London-Queen Square NHS Research Ethics Committee under application number 18/L0/1075.

Provenance and peer review Not commissioned; externally peer reviewed.

Open access This is an open access article distributed in accordance with the Creative Commons Attribution Non Commercial (CC BY-NC 4.0) license, which permits others to distribute, remix, adapt, build upon this work non-commercially, and license their derivative works on different terms, provided the original work is properly cited, appropriate credit is given, any changes made indicated, and the use is non-commercial. See: http://creativecommons.org/licenses/by-nc/4.0/.

\section{ORCID iD}

Veronica Ranieri http://orcid.org/0000-0003-0528-8640

\section{REFERENCES}

1 Lazaridis KN, LaRusso NF. Primary sclerosing cholangitis. N Engl J Med 2016;375:1161-70.

2 Liang H, Manne S, Shick J, et al. Incidence, prevalence, and natural history of primary sclerosing cholangitis in the United Kingdom. Medicine 2017;96:e7116.

3 Molodecky NA, Kareemi H, Parab R, et al. Incidence of primary sclerosing cholangitis: a systematic review and meta-analysis. Hepatology 2011;53:1590-9.
4 Kaplan GG, Laupland KB, Butzner D, et al. The burden of large and small duct primary sclerosing cholangitis in adults and children: a population-based analysis. Am J Gastroenterol 2007;102:1042-9.

5 Lazaridis KN, LaRusso NF. The cholangiopathies. Mayo Clin Proc 2015;90:791-800.

6 Jussila A, Virta LJ, Pukkala E, et al. Malignancies in patients with inflammatory bowel disease: a nationwide register study in Finland. Scand J Gastroenterol 2013;48:1405-13.

7 Ranieri V, McKay K, Walmsley M, et al. Primary sclerosing cholangitis and psychological wellbeing: a scoping review. Semin Liver Dis 2019;39:104-10.

8 Zakharia K, Tabibian A, Lindor KD, et al. Complications, symptoms, quality of life and pregnancy in cholestatic liver disease. Liver Int 2018;38:399-411.

9 Le Strat Y, Le Foll B, Dubertret C. Major depression and suicide attempts in patients with liver disease in the United States. Liver Int 2015;35:1910-6.

10 PSC Support. Clinical need in PSC and clinically meaningful change: what is important to patients. PSC support 2016.

11 PSC Support. Real life PSC. PSC support 2012.

12 Cheung AC, Patel H, Meza-Cardona J, et al. Factors that influence health-related quality of life in patients with primary sclerosing cholangitis. Dig Dis Sci 2016;61:1692-9.

13 Zwaanswijk M, van Dulmen S. Advantages of asynchronous online focus groups and face-to-face focus groups as perceived by child, adolescent and adult participants: a survey study. BMC Res Notes 2014;7:756

14 Williams S, Clausen MG, Robertson A, et al. Methodological reflections on the use of asynchronous online focus groups in health research. Int J Qual Methods 2012;11:368-83.

15 Moodle HQ. Moodle. West Perth, Australia: Moodle HQ, 2018.

16 Blackboard Inc. Blackboard. Washington DC: Blackboard Inc, 2018.

17 Peacock S, Robertson A, Williams S, et al. The role of learning technologists in supporting e-research. ALT-J 2009;17:115-29.

18 QSR International. NVivo. Melbourne, Australia: QSR International, 2018. 